\title{
Effect of aminophylline on fatigue of the sternomastoid muscle in man
}

\author{
J EFTHIMIOU, J FLEMING, RHT EDWARDS, SG SPIRO \\ From the Department of Thoracic Medicine, University College Hospital, London
}

ABSTRACT The effect of intravenous aminophylline on the contractility of the sternomastoid muscle was measured in the fresh state and after the induction of significant fatigue in five normal subjects. Fatigue was produced by repetitive isometric neck flexion, for two seconds every four seconds at $70 \%$ of the maximum voluntary contractile force, continued until exhaustion. Each subject performed three experiments, one to two weeks apart, in random order. In experiment 1 fatiguing exercise and recovery were completed without aminophylline; in experiment 2 intravenous aminophylline was started 30 minutes before exercise and continued throughout the 60 minute recovery period; and in experiment 3 intravenous aminophylline was started immediately after the end of exercise. Aminophylline did not influence the frequency-force relationships, relaxation rate, or maximum voluntary contractile force in the fresh muscle. After fatiguing exercise there was a relatively selective reduction in force response to stimulation frequencies of less than $30 \mathrm{~Hz}$, with little alteration in forces at higher frequencies - that is, low frequency fatigue-and this effect was present for the entire one hour study period. Aminophylline given before or immediately after fatigue did not influence the recovery of either low frequency fatigue or maximum voluntary contractile force. Aminophylline at therapeutic concentrations had no significant effect on the contractility or fatiguability of the normal human sternomastoid muscle.

After strenuous exercise in skeletal muscle a particular type of fatigue develops, which is characterised by a selective reduction of force in response to stimulation frequencies of less than $30 \mathrm{~Hz}$, with little alteration of force response at higher frequencies - that is, low frequency fatigue. This type of fatigue, which is long lasting, was initially described in limb muscles, ${ }^{1}$ but subsequently the same phenomenon was shown to occur in the sternomastoid muscle ${ }^{2}$ and diaphragm $^{3}$ of normal subjects after inspiratory loaded breathing. More recently low frequency fatigue has been demonstrated in the sternomastoid muscle of patients with chronic obstructive lung disease, after a 10 minute period of sustained maximum voluntary ventilation and after a 12 minute walk along a corridor. ${ }^{4}$

Evidence accumulated in the past few years suggests that respiratory muscle fatigue may contribute appreciably to dyspnoea and ventilatory failure, ${ }^{5-7}$ and such fatigue may often be present in patients with

Address for reprint requests: Dr J Efthimiou, Department of Medicine, Rayne Institute, London WCIE $6 \mathrm{JJ}$.

Accepted 30 August 1985 chronic obstructive lung disease. ${ }^{8}$ It has been sug- $\frac{3}{2}$ gested that the therapeutic efficacy of aminophylline in these patients may to a large extent be related to an improvement in respiratory muscle contractility, ${ }^{9}$ and that this could explain the discrepancy that sometimes exists between the poor bronchodilator effect of 0 aminophylline and clinical improvement. ${ }^{1011}$

In isolated limb and respiratory muscle preparations methylxanthine derivatives have been shown to potentiate twitch size and reverse low frequencyo fatigue. ${ }^{12}$ These effects began, however, at concen- $D$ trations of about $1 \mathrm{mmol} / \mathrm{l}$ (equivalent to $180 \mathrm{mg} / \mathrm{l}$ ), which in the case of aminophylline is much too toxic for use in man. ${ }^{1314}$ Recently it has been suggested $O$ that in both man and dog much lower theophyllineN concentrations may improve the diaphragm's con-N tractility and render it less susceptible to fatigue, ${ }^{915} \mathrm{O}$ although this has been disputed. ${ }^{16}$ The potential importance of this controversy for patients in respira-c tory failure led us to investigate the effect of aminophylline on fatigue of the sternomastoid muscle, as it is an important muscle of inspiration in patients witho airflow obstruction and hyperinflation ${ }^{17}$ and is proneo to fatigue. ${ }^{4}$ Furthermore, force production and level

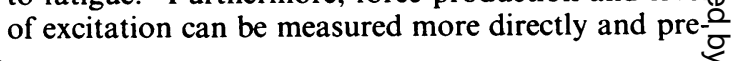


cisely in the sternomastoid muscle than in the diaphragm, particularly during tetanic stimulation. ${ }^{2}$

\section{Methods}

The experiments were performed in five normal subjects, three men and two women, aged 22-32 years, who were volunteers working in the hospital and who had normal lung function. Drinks containing theophylline were avoided for at least 24 hours before each study. All subjects were familiar with the techniques used and gave informed consent. The study was approved by the committee on the ethics of clinical investigation at the School of Medicine, University College London.

A portable apparatus, developed in our laboratory, was used for measuring sternomastoid muscle contraction force and has been described in detail, with modifications, elsewhere. ${ }^{4}$ Unilateral contraction of the sternomastoid muscle produces lateral flexion of the head and neck to the ipsilateral side and rotation to the contralateral side. The force generated by stimulating the right sternomastoid muscle was measured by means of a pressure transducer probe (Dynanometer type-UF2 transducer) with a curved Perspex head, which was positioned to fit around the medial (sternal) tendon of the muscle. The probe displaced the tendon posteriorly and consequently measured an anterior force vector as the muscle contracted and sought to straighten. The probe was kept firmly applied to the muscle tendon by a clamp, and generally resulted in very little discomfort. Subjects were tested with the head and neck in the anatomical position, at $35^{\circ}$ to the horizontal. All exercises and measurements were performed at the same starting sternomastoid muscle length. Breathing was suspended at end tidal expiration and sternomastoid muscle contraction was then stimulated.

The sternomastoid muscle was stimulated electrically with a surface electrode (Red Dot silver/silver chloride self adhesive electrode) placed over its midpoint, where it receives its motor innervation from the spinal accessory nerve. The precise position of the stimulating electrode over the midpoint of the muscle was established by searching for the site at which the maximum force with single stimuli (delivered at $1 \mathrm{~Hz}$ ) was achieved. In this way the sternomastoid muscle was made to contract by stimulation of its intramuscular nerves, with unidirectional square wave impulses of $50 \mu \mathrm{s}$ duration, at $30-60$ volts. The frequency-force curve was generated by stimulating the muscle at set frequencies (that is, 1, 10, 20, 50, and $100 \mathrm{~Hz}$ ) for two seconds in ascending order. This stimulation series was repeated twice on each occasion with a 10 second rest between each series. Supramaximal stimulation of the sternomastoid muscle was not possible in our subjects because of the discomfort it causes, although it was clear that most of the muscle was made to contract vigorously. It has, however, been established in other skeletal muscles that the shape of the frequency-force curve, obtained from maximal stimulation of part of the muscle (as carried out in our studies), is the same as that obtained from supramaximal activation, provided that a substantial proportion of the muscle is made to contract. ${ }^{18}$ As a numerical index of the frequency-force curve the 20:Max ratio was calculated (that is, the ratio of the force generated at $20 \mathrm{~Hz}$ to the maximum force in that stimulation series). After fatiguing exercise this ratio was reduced as the frequency-force curve moved to the right, reflecting low frequency fatigue.

Fatigue of the sternomastoid muscle was produced by repetitive isometric neck flexion for two seconds every four seconds at $70 \%$ of the previously determined maximum voluntary contractile force. This exercise was continued until exhaustion - that is, until the target force, displayed on an oscilloscope, could no longer be achieved on five consecutive attempts. Exhaustion occurred reproducibly after 2.5-3.5 minutes in all five subjects.

Three experiments were performed by each subject, one to two weeks apart, in random order. The protocols for these experiments are shown in figure 1 . Experiments 1 and 2 tested the effect of aminophylline on the frequency-force curve in the fresh muscle, and the ability of the drug to reduce the degree of low frequency fatigue developing in the muscle after exhaustive exericise. Experiment 3 tested the effect of the drug on recovery from fatigue, and for this reason the aminophylline was started immediately after the end of the exercise.

We also wished to discover the time course of recovery of the maximum voluntary contractile force in the sternomastoid muscle after exhaustive exercise, and to ascertain whether intravenous aminophylline affected this in any way. Each subject was therefore asked to produce their maximum voluntary contractile force one, three, six and nine minutes after the fatiguing exercise in each experiment. These values were then compared with the maximum voluntary contractile force produced by the fresh sternomastoid muscle at the beginning of each study. In experiments 1 and 2 the subjects repeated the maximum voluntary contractile force 30 minutes after the baseline tests to determine whether aminophylline had any effect on the maximum voluntary contractile force in the fresh muscle. The relaxation rate of sternomastoid muscle contraction was also measured, as previously described for other skeletal muscles. ${ }^{19}$

In experiment 2 , aminophylline was administered by intravenous infusion into the right arm at a dose of $7 \mathrm{mg} / \mathrm{kg}$ during the first 30 minutes, and subsequently 

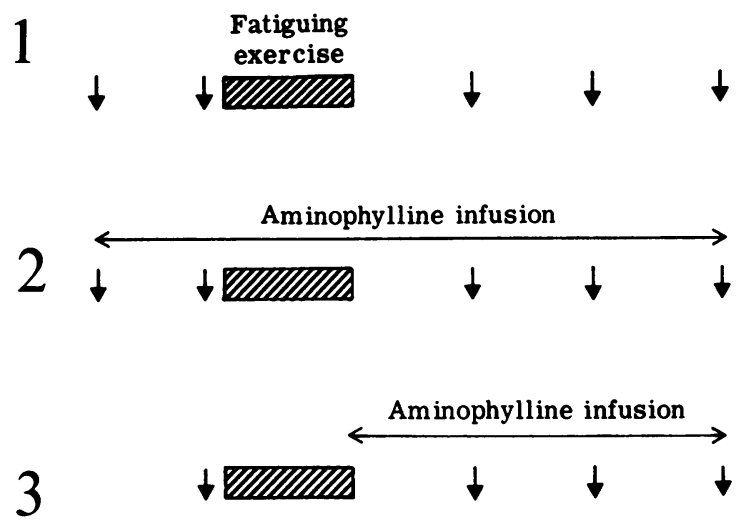

$\begin{array}{lllll}-30 & 0 & 0 & 10 & 30\end{array}$

Time (minutes)

Fig 1 Study protocol (experiments performed in random order). Experiment 1-no aminophylline; experiment 2aminophylline starting before exercise; experiment 3aminophylline starting after exercise. $\downarrow-$ times when frequency-force curves and other measurements were made.

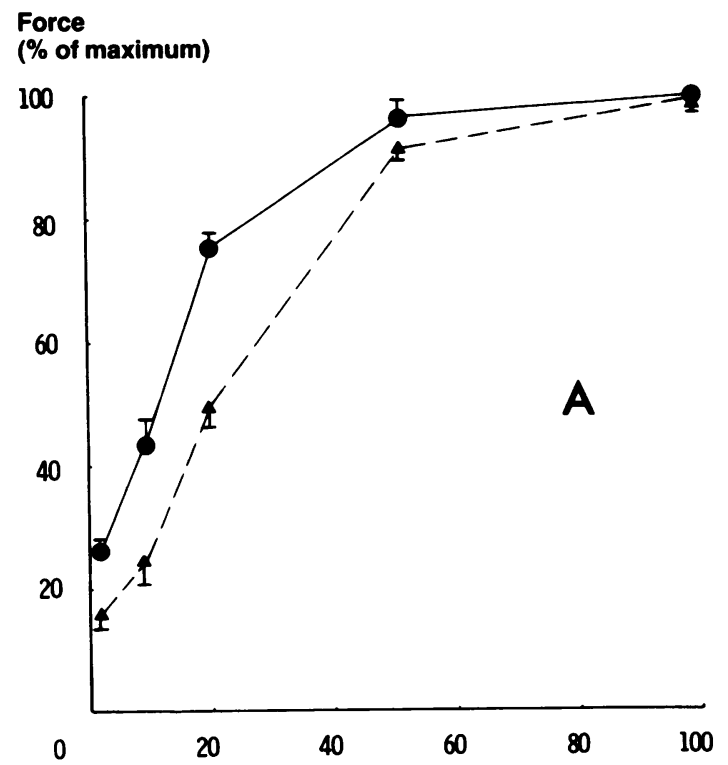

Frequency $(\mathrm{Hz})$ at $1.0 \mathrm{mg} / \mathrm{kg} / \mathrm{h}$ until the end of the study. In experiment 3 aminophylline was administered at a dose of $7 \mathrm{mg} / \mathrm{kg}$ in the first 10 minutes after the fatiguing exercise, and subsequently at $1.0 \mathrm{mg} / \mathrm{kg} / \mathrm{h}$ until the end of the study. Venous blood was taken from the left arm, for estimation of plasma theophylline concentrations, after each pair of frequency-force curves had been obtained. Plasma theophylline concentrations were determined by EMIT homogenous enzyme immunoassay (Syva). All subjects had their electrocardiograms monitored throughout each study.

The percentage fall in 20:Max ratio and maximum voluntary contractile force after fatiguing exercise observed in experiments with and without intra- iv venous aminophylline were compared by use of the Friedman two way analysis of variance.

\section{Results}

Ten (five pairs) control frequency-force curves were $\stackrel{\mathbb{D}}{\mathscr{T}}$ obtained for each subject before they started fatiguing exercise (fig 1) and all were closely similar (fig 2). ₹ These include the two pairs of resting curves obtained $\vec{\bullet}$ in experiment 2 before and after the loading aminophylline infusion. Thirty minutes from the start of experiment 2 there was no shift in the frequency-force curve, the 20:Max ratio (mean (SE)) at the start of experiment 2 being 77.4 (2.0), compared with 76.2 (1.4) 30 minutes later.

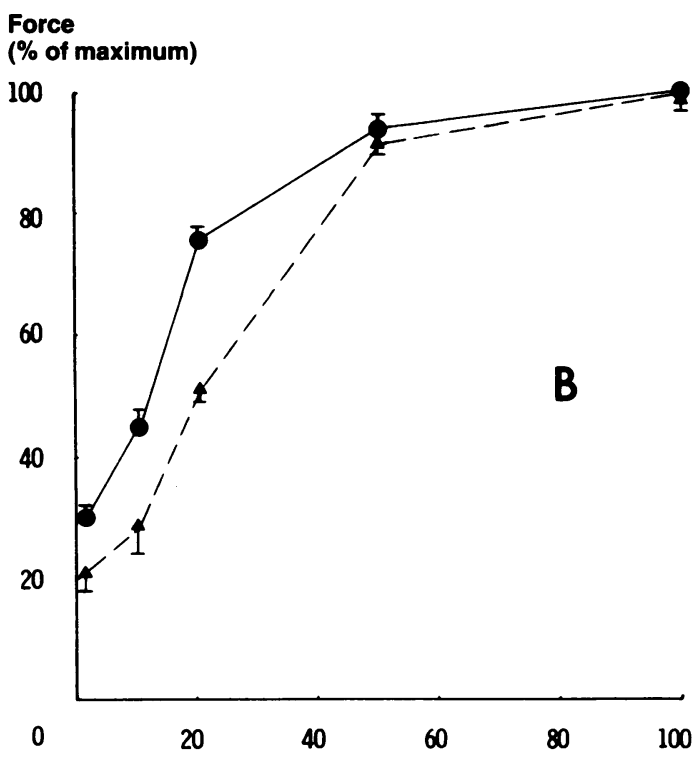

Frequency $(H z)$

Fig 2 Mean frequency-force curves in five subjects before fatigue (O) and 10 minutes after fatigue $(\boldsymbol{\Delta}):(A)$ data obtained in the absence of aminophylline (experiment 1 ); (B) curves obtained with aminophylline (experiment 2). Force is expressed as a percentage of the maximum force produced in that stimulation series. Results are means with standard errors. 
The exercise protocol produced substantial low frequency fatigue as well as significant reductions in the maximum voluntary contractile force in all subjects. The mean maximum falls in 20:Max ratio (expressed as a percentage of the baseline values) 10 minutes after fatigue were $36.7 \%(3.2 \%), 34.6 \%(1.9 \%)$, and $33.7 \%(2.8 \%)$ in experiments 1,2 (fig 2 ), and 3 respectively. There were no significant differences in the percentage falls in 20:Max ratio between experiments with and without intravenous aminophylline infusion at 10,30 , or 60 minutes after fatigue, in any of the five subjects (fig 3). Aminophylline had no obvious effect on the extent of reduction in force at any frequency at any time during the experiments.

The maximum fall in maximum voluntary contractile force was seen at one minute after fatigue with mean maximum percentage falls in force of $24.3 \%$ $(4.8 \%), 23.7 \%(2.7 \%)$, and $24.4 \%(4.2 \%)$ for experiments 1,2 (fig 4), and 3 respectively. The mean baseline maximum voluntary contractile forces were 7.9 $(1.0) \mathrm{kg}, 8.1(1.2) \mathrm{kg}$, and $8.1(1.3) \mathrm{kg}$ in experiments 1 , 2 (fig 4), and 3 respectively and all returned to within $10 \%$ of the baseline value nine minutes after the end of fatiguing exercise (fig 4). Aminophylline had no discernible effect on the maximum fall in or recovery of the maximum voluntary contractile force after exhaustive exercise in any subject. Relaxation rates in the fresh sternomastoid muscle were not affected by aminophylline infusion in experiment 2 , and on subsequent occasions after the onset of fatigue when the frequency-force measurements were made relaxation rates were not significantly different from their baseline values in any of the experiments.
In experiment 2 the mean plasma theophylline concentrations for the five subjects immediately before and 10 minutes after fatiguing exercise were 15.2 (range 14.1-16.9) $\mathrm{mg} / \mathrm{l}$ and 16.2 (range $14.8-17.3) \mathrm{mg} / \mathrm{l}$ respectively. In experiment 3 the mean plasma theophylline concentration 10 minutes after fatiguing exercise was 15.0 (range $13.2-17.2) \mathrm{mg} / \mathrm{l}$. In both experiments these concentrations were maintained throughout the 60 minute study period (table).

\section{Discussion}

These results suggest that aminophylline at concentrations known to be therapeutically acceptable in man had no obvious potentiating effect on the fresh or fatigued sternomastoid muscle. The experimental protocol resulted in appreciable low frequency fatigue of the sternomastoid muscle, as indicated by a shift in the frequency-force curve to the right with reduction of the 20:Max ratio, which persisted throughout the study period. Neither the development of this fatigue nor recovery from it was influenced by concentrations of aminophylline in the upper half of the normal therapeutic range. The maximum voluntary contractile force produced by the fresh muscle was also unaffected by aminophylline, and its recovery during the first nine minutes after exhaustive exercise was not improved. High motor neurone firing frequencies (that is, $>30 \mathrm{~Hz}$ ) are necessary to produce maximum voluntary contractions, ${ }^{20}$ and such frequencies can be maintained for only short periods. Aminophylline seems unlikely therefore to have any major effect on

\section{Force at $20 \mathrm{~Hz}$}

(\% of maximum)

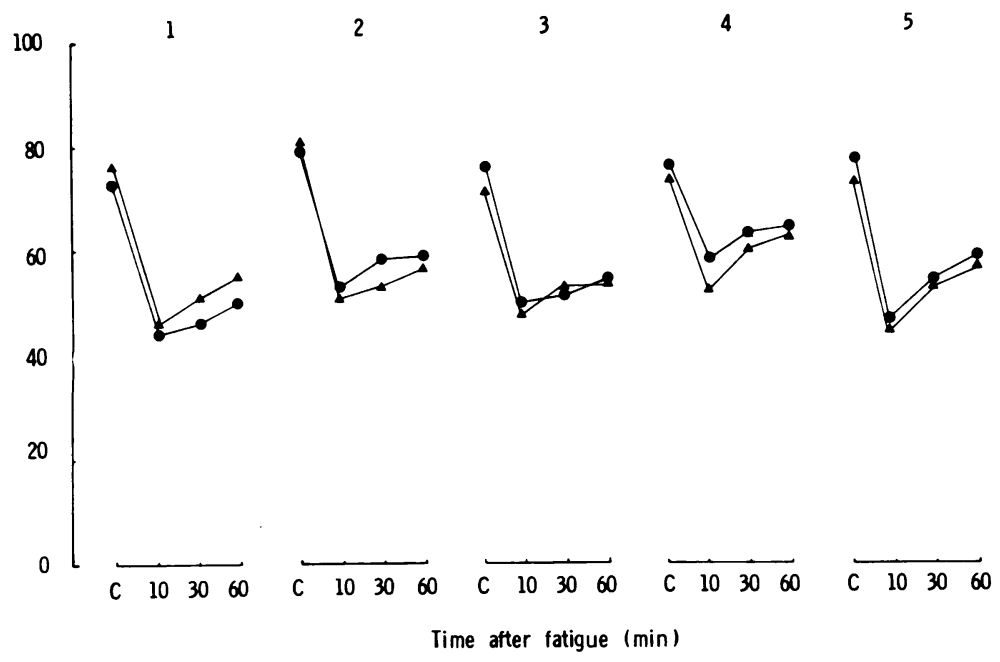

Fig 3 Effect of intravenous aminophylline on recovery of low frequency fatigue. 20:Max ratios are shown before (C) and 10,30, and 60 minutes after fatigue in five subjects. -control series, no aminophylline (experiment 1); $\Delta$-aminophylline series (experiment 2). 
recovery of high frequency fatigue either. It is possible that a small effect of the drug has not been detected, but an important potentiating action is unlikely to have been missed in view of the relatively small variability of the data (fig 2).

In isolated limb and respiratory muscle preparations methylxanthine derivatives, in relatively high concentrations, have been shown to potentiate twitch size and reverse low frequency fatigue. ${ }^{1221}$ Recently

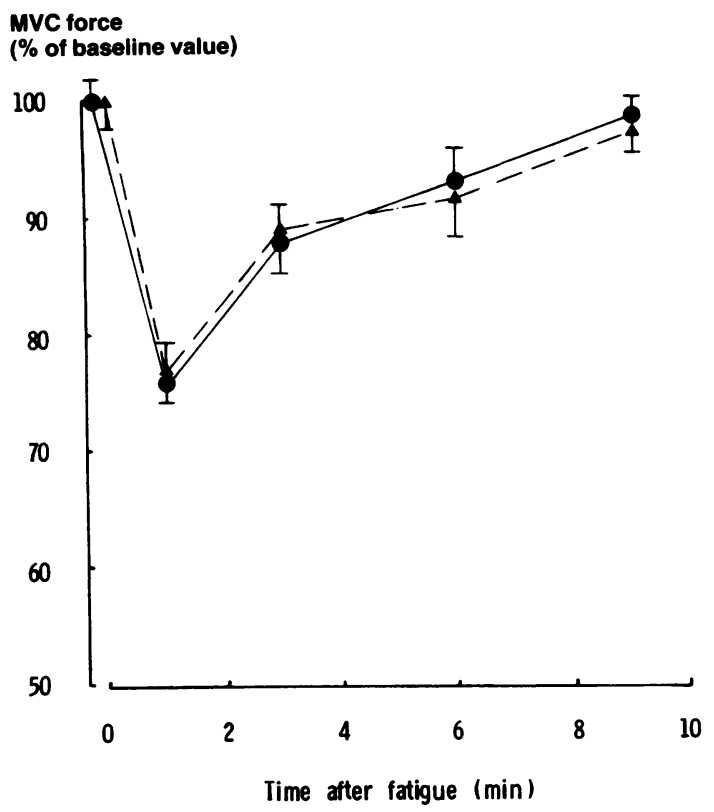

Fig 4 Effect of intravenous aminophylline on recovery of the maximum voluntary contractile (MVC) force after fatigue. -control series, no aminophylline (experiment 1); $\Delta$-aminophylline series (experiment 2). Maximum voluntary contractile force is expressed as a percentage of the baseline value before fatigue. Results are means with standard errors for five subjects. it has been suggested that aminophylline affects calcium channels on the muscle cell membrane, and that its effect in improving diaphragm contractility depends on the presence of extracellular calcium. ${ }^{22}$ The actions of methylxanthines on skeletal muscle may, however, depend not only on the concentration used but also on the type of muscle- that is, whether it is of the fast or the slow twitch type. Significant potentiation of twitch size in isolated preparations begins at levels of around $1 \mathrm{mmol} / \mathrm{l}^{12} \mathrm{In}$ man, however, toxicity is often a serious problem even at concentrations of less than $0.2 \mathrm{mmol} / \mathrm{l}$ (that is,

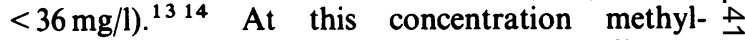
xanthines are likely to have at best a minimal effect on iv skeletal muscle, ${ }^{12}$ and it is therefore difficult to know $\vec{N}$ whether the benefit to patients taking aminophylline $N$

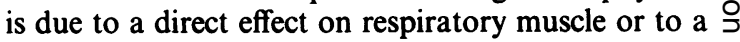
consequence of some of the other actions of the drug, $\overrightarrow{-}$ such as bronchodilatation, central nervous system stimulation, increasing cardiac output, vasodilation, or mobilisation of free fatty acids. ${ }^{21}$

In clinical practice low frequency fatigue has been demonstrated in the respiratory muscles of patients $\vec{\theta}$ with chronic obstructive lung disease, ${ }^{4}$ and it has been suggested that such patients may benefit from 0 infusions of aminophylline because of improved contractility of the diaphragm and consequent increased resistance to fatigue. ${ }^{9}$ We found no evidence, however, of a beneficial effect of aminophylline on the sternomastoid muscle in the fresh or fatigued state, despite good therapeutic concentrations of the drug. Our findings are supported by the study of Wiles et al, ${ }^{16}$ who found that aminophyline had no significant effect on adductor pollicis contractility or fatiguability in normal subjects. It is important perhaps to emphasise here that force production and level of excitation can be measured more directly and precisely in the adductor pollicis and sternomastoid muscles than in the diaphragm, particularly during tetanic stimulation. ${ }^{2}$

The possibility remains that the diaphragm is more

Plasma theophylline concentrations ( $\mathrm{mg} / \mathrm{l}$ ) before and after fatigue in five normal subjects

\begin{tabular}{|c|c|c|c|c|c|c|c|c|c|c|c|}
\hline \multirow{3}{*}{$\begin{array}{l}\text { Subject } \\
\text { No }\end{array}$} & \multicolumn{6}{|c|}{ Experiment 2} & \multicolumn{5}{|l|}{ Experiment 3} \\
\hline & \multicolumn{2}{|c|}{ Min before fatigue } & \multicolumn{4}{|c|}{ Min after fatigue } & \multirow{2}{*}{$\frac{\text { Min before fatigue }}{0}$} & \multicolumn{4}{|c|}{ Min after fatigue } \\
\hline & 0 & 30 & 10 & 30 & 60 & Mean & & 10 & 30 & 60 & Mean \\
\hline $\begin{array}{l}1 \\
2 \\
3 \\
4 \\
5\end{array}$ & $\begin{array}{l}0 \\
0 \\
0 \\
0 \\
0 \\
\mathbf{M}\end{array}$ & $\begin{array}{l}14.1 \\
14.8 \\
16.9 \\
14.2 \\
15.8 \\
\text { for gro }\end{array}$ & $\begin{array}{r}16.7 \\
14.8 \\
17.3 \\
15.7 \\
16.4 \\
\text { n expe }\end{array}$ & $\begin{array}{r}17.5 \\
16.2 \\
17.8 \\
19.7 \\
17.1 \\
\text { ent } 2 \text { : }\end{array}$ & $\begin{array}{r}17.9 \\
15.7 \\
19.1 \\
20.8 \\
17.7 \\
8(0.6)\end{array}$ & $\begin{array}{l}16.6 \\
15.4 \\
17.8 \\
17.6 \\
16.8\end{array}$ & $\begin{array}{l}0 \\
0 \\
0 \\
0 \\
0 \\
\text { Mean (SE) for gro }\end{array}$ & $\begin{array}{l}13.7 \\
16.1 \\
17.2 \\
15.0 \\
13.2 \\
\text { n expe }\end{array}$ & $\begin{array}{r}13.9 \\
17.6 \\
17.9 \\
18.0 \\
15.3 \\
\text { ent } 3:\end{array}$ & $\begin{array}{l}14.8 \\
16.4 \\
17.9 \\
17.1 \\
16.8 \\
(0.8)\end{array}$ & $\begin{array}{l}14.1 \\
16.7 \\
17.1 \\
16.7 \\
15.1\end{array}$ \\
\hline
\end{tabular}


sensitive to aminophylline than either the sternomastoid or the adductor pollicis muscles, although the in vitro frequency-force curves, the development of low frequency fatigue, and the response to high concentrations of theophylline (that is, $\geqslant 1 \mathrm{mmol} / \mathrm{l}$ ) appear to be the same in limb muscles as they are in the sternomastoid muscle and diaphragm. ${ }^{12}$

The discrepancy between the results of our study and some of the previously published data ${ }^{915}$ is not easily explained, although differences in experimental protocols, differences in the sensitivity of different muscles to aminophylline, and also species differences may all play a part. Further human studies, perhaps of a collaborative nature, may help to resolve this controversy.

We conclude that the therapeutic benefit of aminophylline in man is unlikely to be due to an effect on the contractility of the respiratory muscles. Agents that potentiate skeletal muscle contractility could be effective in counteracting respiratory muscle fatigue, but a clinically useful compound would need to be much more potent than the methyl xanthine drugs currently in use.

We are grateful to Dr H Gribbin for his helpful advice in preparing this manuscript and to Anne Blakeney in the Department of Chemical Pathology for performing the aminophylline assays. This work was supported by a grant from the North East Thames Regional Health Authority Research Council. The manuscript was prepared with great care by Angela Betchley.

\section{References}

1 Edwards RHT, Hill DK, Jones DA, Merton PA. Fatigue of long duration in human skeletal muscle after exercise. J Physiol (Lond) 1977;272:769-78.

2 Moxham J, Wiles CM, Newham D, Edwards RHT. Sternomastoid muscle function and fatigue in man. Clin Sci 1980;59:463-8.

3 Moxham J, Morris AJR, Spiro SG, Edwards RHT, Green M. Contractile properties and fatigue of the diaphragm in man. Thorax 1981;36:164-8.

4 Wilson SH, Cooke NT, Moxham J, Spiro SG. Sternomastoid muscle function and fatigue in normal subjects and in patients with chronic obstructive pulmonary disease. Am Rev Respir Dis 1984;129:460-4.

5 Roussos C, Macklem PT. Diaphragmatic fatigue in man. J Appl Physiol 1977;43:189-97.
6 Macklem PT, Roussos C. Respiratory muscle fatigue: a cause of respiratory failure? Clin Sci Mol Med 1977; 53:419-22.

7 Roussos C, Fixley M, Gross D, Macklem PT. Fatigue of inspiratory muscles and their synergistic behaviour. $J$ Appl Physiol 1979;46:897-904.

8 Grassino A, Gross D, Macklem PT, Roussos C, Zagelbaum G. Inspiratory muscle fatigue as a factor limiting exercise. Bull Eur Physiopathol Respir 1979;15:105-11.

9 Aubier M, De Troyer A, Sampson M, Macklem PT, Roussos C. Aminophylline improves diaphragmatic contractility. N Engl J Med 1981;305:249-52.

10 Estenne M, Yernault JC, De Troyer A. Effects of parenteral aminophylline on lung mechanics in normal humans. Am Rev Respir Dis 1980;121:967-71.

11 Rossing TH, Fanta CH, Goldstein DH, Snapper JR, McFadden ER jun. Emergency therapy of asthma: comparison of the acute effects of parenteral and inhaled sympathomimetics and infused aminophylline. Am Rev Respir Dis 1980;122:365-71.

12 Jones DA, Howell S, Roussos C, Edwards RHT. Low frequency fatigue in isolated skeletal muscles and the effects of methylxanthines. Clin Sci 1982;63:161-7.

13 Zwillich CW, Sutton FD, Neff TA, et al. Theophylline induced seizures in adults, correlation with serum concentrations. Ann Intern Med 1976;82:784-7.

14 Kordash TR, Van Dellen RG, McCall JT. Theophylline concentrations in asthmatic patients after administration of aminophylline. JAMA 1977;238:139-41.

15 Sigrist S, Thomas D, Howell S, Roussos C. The effect of aminophylline on inspiratory muscle contractility. Am Rev Respir Dis 1982;126:46-60.

16 Wiles CM, Moxham J, Newham D, Edwards RHT. Aminophylline and fatigue of adductor pollicis in man. Clin Sci 1983;64:547-50.

17 Skarvan K, Mikulenka V. The ventilatory function of sternomastoid and scalene muscles in patients with pulmonary emphysema. Respiration 1970;27:480-92.

18 Edwards RHT, Young A, Hosking GP, Jones DA. Human skeletal muscle function: description of tests and normal values. Clin Sci 1977;63:282-90.

19 Wiles CM, Young A, Jones DA, Edwards RHT. Muscle relaxation rate, fibre-type composition and energy turnover in hyper- and hypo-thyroid patients. Clin Sci 1979;57:375-84.

20 Jones DA, Bigland-Ritchie B, Edwards RHT. Excitation frequency and muscle fatigue: mechanical responses during voluntary and stimulated contractions. Exp Neurol 1979;64:401-13.

21 Rall TW. Central nervous system stimulants: the xanthines. In: Gilman AG, Goodman LS, Gilman A, eds. The pharmacological basis of therapeutics. 6th ed. New York: Macmillan, 1980:592-607.

22 Aubier M, Marciano D, Viires N, Lecocguic Y, Pariente R. Diaphragmatic contractility enhanced by aminophylline: role of extracellular calcium. J Appl Physiol: Respir Environ Exercise Physiol 1983;54:460-4. 